\title{
The narrow ridge from liver damage to hepatocarcinogenesis
}

\author{
Jan Best ${ }^{1}$, Guido Gerken ${ }^{2}$ \\ 'Department of Internal Medicine, University Hospital Knappschaftskrankenhaus Bochum, Ruhr University Bochum, Bochum \\ 44892, Germany. \\ ${ }^{2}$ Department of Gastroenterology and Hepatology, University Hospital Essen, Essen 45147, Germany.
}

Correspondence to: Dr. Jan Best, MD, Department of Internal Medicine, University Hospital Knappschaftskrankenhaus Bochum, In der Schornau 23-25, Bochum 44892, Germany. E-mail: jan.best@kk-bochum.de

How to cite this article: Best J, Gerken G. The narrow ridge from liver damage to hepatocarcinogenesis. Hepatoma Res $2021 ; 7: 32$. https://dx.doi.org/10.20517/2394-5079.2021.52

Received: 13 Apr 2021 Accepted: 14 Apr 2021 Published: 17 Apr 2021

Academic Editor: Guang-Wen Cao Copy Editor: Xi-Jun Chen Production Editor: Xi-Jun Chen

Globally, hepatocellular carcinoma (HCC) is associated with a poor prognosis due to frequent diagnosis in late stages. As a result, mortality is roughly equivalent to the annual incidence. Regardless of etiology, the development of liver cirrhosis is the most relevant cause of hepatocarcinogenesis ${ }^{[1]}$. Chronic hepatitis B virus (HBV) infection and nonalcoholic steatohepatitis (NASH) are associated with a significantly increased risk of developing HCC even in the absence of cirrhosis $^{[2]}$. Recent data demonstrate that NASH has to be considered as the main cause of the increasing incidence of HCC predicted for the next decades ${ }^{[3]}$. Thus, against the background of high mortality, the need for optimized HCC screening strategies arises on the one hand, while, on the other hand, previous treatment concepts have to be adapted to the sometimes therapylimiting comorbidities of these patients.

The global prevalence of non-alcoholic fatty liver disease (NAFLD) is estimated to be $25 \%$, although this figure may still be underestimated due to an assumed high number of unreported cases ${ }^{[4]}$. In approximately $10 \%-15 \%$ of NAFLD patients, progression to NASH can occur, which in turn can lead to higher-grade fibrosis and, in the final stage, to cirrhosis and $\mathrm{HCC}^{[5]}$. Approximately $20 \%$ of all NASH-associated HCC cases develop in the absence of overt cirrhosis $^{[6]}$. According to the most recent epidemiological studies, NASH is considered as the main cause of the steadily increasing incidence of HCC in Western industrialized nations ${ }^{[7]}$. Recent data from a United States cohort show a 10-year cumulative HCC risk of 1.7 per 1000 NAFLD patients. In NASH patients, HCC risk was increased 8.6-fold compared with healthy subjects. Patients with NAFLD-associated cirrhosis showed a further significantly increased risk of HCC, 
with an annual incidence of $0.8 \%-2.3 \%$ per year. Of note, prevalence of type II diabetes mellitus (T2DM) and obesity appear to be independent HCC-predisposing risk factors in the context of NASH-associated metabolic syndrome. Furthermore, in NASH patients, initial HCC diagnosis is frequently made at later tumor stages because they are monitored less intensively due to the absence of, or less advanced, cirrhosis ${ }^{[8]}$.

Human hepatocarcinogenesis is always a multifactorial event. Environmental influences (e.g., alcohol and smoking) and host factors (age, gender, familial predisposition, obesity, diabetes, and fibrosis) interact with genetic, immunological, and viral factors in a complex interplay in HCC development. In the context of DNA sequence changes in the form of mutations, resistance of proliferating hepatocytes to programmed cell death (apoptosis) occurs, allowing mutant cells to proliferate ${ }^{[0]}$ [Figure 1]. In addition, in NASH, chronic hepatic inflammation represents a cirrhosis-independent trigger of hepatocarcinogenesis. It is a complex, multifactorial process involving various risk factors (genomic instability, obesity, and T2DM). An understanding of the various key molecular initiation and progression mechanisms of hepatocarcinogenesis is essential to define etiology-adapted therapeutic targets in the future.

Routine ultrasound (US) of the liver for HCC screening exhibits distinct limitations in terms of diagnostic value. Smaller lesions show limited detectability in the cirrhotically remodeled or steatotic liver. Determination of HCC biomarkers, such as alpha-fetoprotein (AFP), has the advantage of being investigator independent and to provide reproducible results. However, not all international guidelines recommend AFP determination in addition to US, since sensitivity of AFP in HCC detection ranges from $39 \%$ to $65 \%$ only ${ }^{[10]}$. To facilitate sensitivity superior to the combination of US and AFP while maintaining good specificity in early HCC detection, a large number of studies investigated the suitability of different biomarkers, such as osteopontin, glypican-3, the AFP variant binding to the lectin molecule "Lens culinaris agglutinin" (AFP-L3), and Des-gamma-carboxy prothrombin (DCP) for HCC surveillance ${ }^{[1-14]}$. As s result, a statistics-based model called GALAD score, including patients gender, age, AFP, AFP-L3, and DCP, was developed. It clearly demonstrated superior sensitivity and specificity compared to assessment of biomarkers individually and was extensively validated in international studies ${ }^{[15,16]}$. In addition, GALAD scores' utility in HCC early detection was specifically tested in an international multicenter trial in a NASH population; it was able to detect HCC even at early stages (BCLC 0/A) and achieved an AUC of 0.91. Furthermore, in a prospective NASH pilot trial, it could be demonstrated that GALAD was already elevated up to 1.5 years before the initial diagnosis of HCC was made by imaging procedures. Those encouraging results implicate that the GALAD model is suitable for HCC early detection of all etiologies, including $\mathrm{NASH}^{[17]}$. Only earlier HCC detection, predominantly in the emerging high-risk cohort of patients with NASH, T2DM, and obesity, can facilitate curative treatment options for this population. Further phase IV prospective trials are urgently warranted to determine whether GALAD has potential as an integral part of HCC screening algorithms in the near future.

Currently, HCCs are frequently diagnosed at advanced stages when curative therapy options such as liver resection or transplantation are unavailable. In addition to established palliative, locoregional therapies, such as conventional transarterial chemoembolization (TACE), alternative transarterial procedures such as selective internal radiotherapy (SIRT) are also available [Figure 2]. Recent randomized clinical trials (RCT) showed no survival benefit for SIRT over systemic therapy in patients with advanced $\mathrm{HCC}^{[18]}$. A metaanalysis on the latest RCTs in contrast could demonstrate that SIRT is non-inferior to standard of care systemic therapy in terms of overall survival (OS) while offering an adequate safety profile with fewer side effects. Furthermore, patients with advanced HCCs arising in NAFLD may not be suitable for systemic therapy due to metabolic syndrome-related diseases. Here, SIRT exhibited similar survival outcomes at a comparable toxicity profile in HCC patients with NAFLD and HBV, implicating that NAFLD-associated 


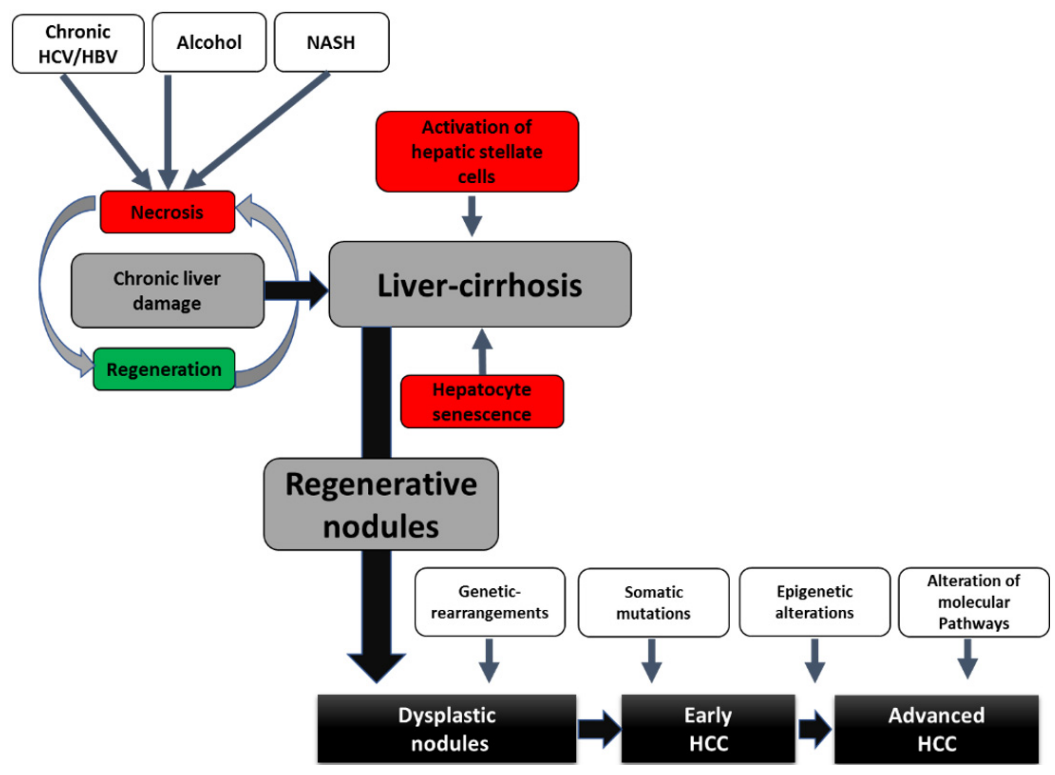

Figure 1. Progression from chronic liver injury to HCC. Persistent exposure of the liver to harmful stimuli (viral hepatitis, alcohol abuse, and NASH) induces necrosis of the hepatocytes and thus initiates compensatory regenerative processes. This leads to hepatocytebased regeneration, a vicious circle of hepatocyte senescence and hepatic stellate cell activation with deposition of interstitial matrix and consecutive fibrosis formation up to liver cirrhosis occurs. The resulting genomic instability initiates the cascade of hepatocarcinogenesis from dysplastic nodules to the development of HCC. A stepwise accumulation of multiple events such as genetic rearrangements, somatic mutations, epigenetic alterations, and activation of various growth factor signaling pathways leads to tumor proliferation and metastasis. HCV: Hepatitis C virus; HBV: hepatitis B virus; HCC: hepatocellular carcinoma.

metabolic comorbidities do not represent limitations for SIRT.

Advanced HCCs at stage BCLC C, ineligible for surgical or local therapy due to vascular infiltration or extrahepatic tumor burden, have to be treated with systemic therapy. Only recently, several substances over three lines of therapy have become available [Figure 3]. In 2007, the multityrosine kinase inhibitor sorafenib was approved for the treatment of advanced HCC in the phase III SHARP trial; here, for the first time, an antiproliferative therapy was able to significantly prolong the OS compared to placebo treatment (OS sorafenib 10.7 months $v$ s. placebo 7.9 months $)^{[19]}$.

The first substance to show a significant survival benefit in a phase III second-line trial after sorafenib (RESORCE) was the tyrosine kinase inhibitor (TKI) regorafenib for those patients tolerant to prior sorafenib treatment (OS after progression on sorafenib: regorafenib 10.6 months vs. placebo 7.8 months) ${ }^{[20]}$. Shortly thereafter, in mid-2017, data from a Phase III study were published in which the TKI lenvatinib was tested against sorafenib in first-line treatment of HCC (REFLECT study). It showed non-inferiority to sorafenib with equivalence of OS (lenvatinib 13.6 months $v s$. sorafenib 12.3 months) ${ }^{[21]}$. Lenvatinib showed superiority on secondary endpoints, such as progression-free survival, with comparable toxicity profile. Subsequently, in 2018, results from a phase III study were published, in which the TKI cabozantinib vs. placebo as second-line treatment of HCC (CELESTIAL) significantly prolonged overall survival (OS after progression on sorafenib: cabozantinib 10.2 months $v$ s. placebo 8.0 months $)^{[22]}$.

However, in terms of clinical management, there are distinct safety concerns for several patients, particularly those who have cardiovascular disease in the setting of NASH-associated metabolic syndrome. In particular, typical TKI side effects, such as poorly controlled arterial hypertension, fatigue, weight loss, hand-foot-skin syndrome, and diarrhea, often lead to the need for dose reduction or therapy interruption. A 


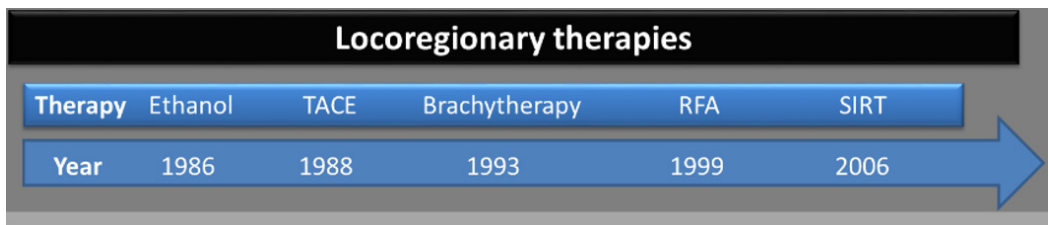

\section{Systemic treatments}

\begin{tabular}{|c|c|c|c|}
\hline \multicolumn{4}{|l|}{ First line } \\
\hline Therapy & Sorafenib & Lenvatinib & Atezolizumab/Bevacizumab \\
\hline Year & 2007 & 2018 & 2020 \\
\hline OS months & 10.7 & 13.9 & 19.2 \\
\hline \multicolumn{4}{|c|}{ Second line } \\
\hline Therapy & Regorafenib & Cabozantinib & Ramucirumab \\
\hline Year & 2017 & $\frac{1}{2018}$ & 2019 \\
\hline real & 2011 & 20 & 2019 \\
\hline OS months & 10.6 & 10.2 & 8.1 \\
\hline
\end{tabular}

Figure 2. Evolution of locoregionary and systemic HCC treatment options. Initially, no systemic HCC treatments were available, and the numerous locoregionary therapies either percutaneously (ethanol injection, brachytherapy or radiofrequency ablation) or transcatheter-based methods (TACE or SIRT) were the only alternatives, when liver resection or transplantation were not feasible. In 2007, sorafenib was granted approval as systemic first line HCC treatment. After almost a decade, different first and second line TKI treatment options evolved. Ramucirumab, an anti-VEGFR-2 monoclonal antibody, was the only non-TKI-based systemic second-line treatment available until 2020. Then, for the first, time an immune checkpoint-inhibitor-based treatment (atezolizumab combined with bevacizumab) was granted approval in HCC first line treatment. TACE: Transarterial chemoembolization; SIRT: selective internal radiotherapy; RFA: radiofrequency ablation; OS: overall survival; TKI: tyrosine kinase inhibitor.

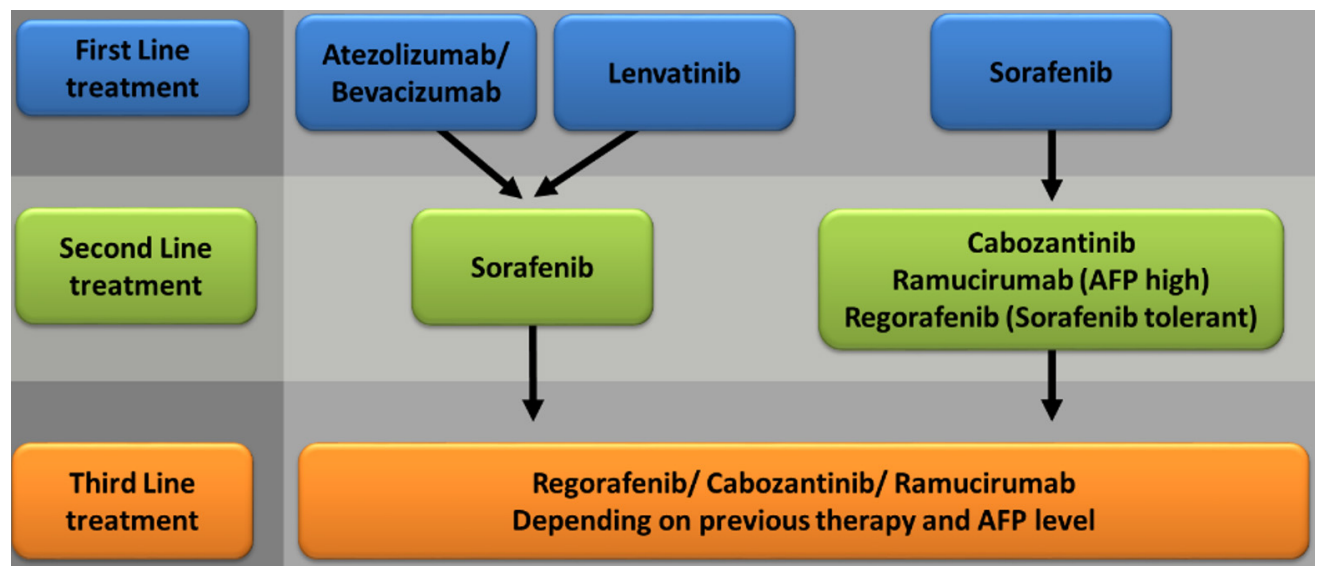

Figure 3. Systemic treatment algorithm proposed by the authors. Therapeutical regimens should be selected in adaption to patients' comorbidities, liver function, tumor burden/localization, AFP level, and tolerance to previous systemic treatment. AFP: Alphafetoprotein.

non-TKI-based treatment option for a subset of HCC patients with an elevated AFP-level $(\geq 400 \mathrm{ng} / \mathrm{mL})$ is ramucirumab, a fully human anti-VEGFR-2 monoclonal antibody. In a phase III second-line study after sorafenib progression (REACH-II study), ramucirumab showed a significantly prolonged OS in patients with high AFP (OS after progression on sorafenib: ramucirumab 8.5 months vs. placebo 7.3 months) ${ }^{[23]}$. 
Immunotherapy of tumors with immune checkpoint inhibitors has also shown encouraging results in a variety of tumor diseases. In a phase III study (IMbrave150), the combination of a checkpoint inhibitor (atezolizumab) with a VEGF antibody (bevacizumab) compared with sorafenib treatment significantly improved OS in first-line HCC therapy of HCC $^{[24]}$. Furthermore, a better quality of life was documented compared to sorafenib. Thus, this was the first positive phase III study to show an OS benefit in favor of cancer immunotherapy in this tumor entity and represents the first landmark therapeutic advance in more than a decade.

\section{DECLARATIONS}

\section{Authors' contributions}

Conception or design of the work, drafting the article: Best J

Critical revision of the article, final approval of the version to be published: Best J, Gerken G

\section{Availability of data and materials}

Not applicable.

\section{Financial support and sponsorship}

None.

\section{Conflicts of interest}

Both authors declared that there are no conflicts of interest.

\section{Ethical approval and consent to participate}

Not applicable.

\section{Consent for publication}

Not applicable.

\section{Copyright}

(c) The Author(s) 2021.

\section{REFERENCES}

1. Fujiwara N, Qian T, Koneru B, Hoshida Y. Omics-derived hepatocellular carcinoma risk biomarkers for precision care of chronic liver diseases. Hepatol Res 2020;50:817-30. DOI PubMed

2. Ertle J, Dechêne A, Sowa JP, et al. Non-alcoholic fatty liver disease progresses to hepatocellular carcinoma in the absence of apparent cirrhosis. Int J Cancer 2011;128:2436-43. DOI PubMed

3. Wong SW, Ting YW, Chan WK. Epidemiology of non-alcoholic fatty liver disease-related hepatocellular carcinoma and its implications. JGH Open 2018;2:235-41. DOI PubMed PMC

4. Araújo AR, Rosso N, Bedogni G, Tiribelli C, Bellentani S. Global epidemiology of non-alcoholic fatty liver disease/non-alcoholic steatohepatitis: what we need in the future. Liver Int 2018;38 Suppl 1:47-51. DOI PubMed

5. Vernon G, Baranova A, Younossi ZM. Systematic review: the epidemiology and natural history of non-alcoholic fatty liver disease and non-alcoholic steatohepatitis in adults. Aliment Pharmacol Ther 2011;34:274-85. DOI PubMed

6. Kanwal F, Kramer JR, Mapakshi S, et al. Risk of hepatocellular cancer in patients with non-alcoholic fatty liver disease. Gastroenterology 2018;155:1828-37.e2. DOI PubMed PMC

7. Younossi Z, Stepanova M, Ong JP, et al; Global Nonalcoholic Steatohepatitis Council. Nonalcoholic steatohepatitis is the fastest growing cause of hepatocellular carcinoma in liver transplant candidates. Clin Gastroenterol Hepatol 2019;17:748-55.e3. DOI PubMed

8. Weinmann A, Alt Y, Koch S, et al. Treatment and survival of non-alcoholic steatohepatitis associated hepatocellular carcinoma. BMC Cancer 2015;15:210. DOI PubMed PMC

9. Dhanasekaran R, Bandoh S, Roberts LR. Molecular pathogenesis of hepatocellular carcinoma and impact of therapeutic advances. F1000Res 2016;5:F1000 Faculty Rev-879. DOI PubMed PMC

10. Daniele B, Bencivenga A, Megna AS, Tinessa V. Alpha-fetoprotein and ultrasonography screening for hepatocellular carcinoma. Gastroenterology 2004;127:S108-12. DOI PubMed

11. Shang S, Plymoth A, Ge S, et al. Identification of osteopontin as a novel marker for early hepatocellular carcinoma. Hepatology 
2012;55:483-90. DOI PubMed PMC

12. Wang XY, Degos F, Dubois S, et al. Glypican-3 expression in hepatocellular tumors: diagnostic value for preneoplastic lesions and hepatocellular carcinomas. Hum Pathol 2006;37:1435-41. DOI PubMed

13. Liebman HA, Furie BC, Tong MJ, et al. Des-gamma-carboxy (abnormal) prothrombin as a serum marker of primary hepatocellular carcinoma. N Engl J Med 1984;310:1427-31. DOI PubMed

14. Li D, Mallory T, Satomura S. AFP-L3: a new generation of tumor marker for hepatocellular carcinoma. Clin Chim Acta 2001;313:159. DOI PubMed

15. Johnson PJ, Pirrie SJ, Cox TF, et al. The detection of hepatocellular carcinoma using a prospectively developed and validated model based on serological biomarkers. Cancer Epidemiol Biomarkers Prev 2014;23:144-53. DOI PubMed

16. Berhane $\mathrm{S}$, Toyoda $\mathrm{H}$, Tada $\mathrm{T}$, et al. Role of the GALAD and BALAD-2 serologic models in diagnosis of hepatocellular carcinoma and prediction of survival in patients. Clin Gastroenterol Hepatol 2016;14:875-86.e6. DOI PubMed

17. Best J, Bechmann LP, Sowa JP, et al. GALAD score detects early hepatocellular carcinoma in an international cohort of patients with nonalcoholic steatohepatitis. Clin Gastroenterol Hepatol 2020;18:728-35.e4. DOI PubMed

18. Venerito M, Pech M, Canbay A, et al. NEMESIS: noninferiority, individual-patient metaanalysis of selective internal radiation therapy with (90)y resin microspheres versus sorafenib in advanced hepatocellular carcinoma. J Nucl Med 2020;61:1736-42. DOI PubMed

19. Llovet JM, Ricci S, Mazzaferro V, et al; SHARP Investigators Study Group. Sorafenib in advanced hepatocellular carcinoma. $N$ Engl J Med 2008:359:378-90. DOI PubMed

20. Bruix J, Qin S, Merle P, et al; RESORCE Investigators. Regorafenib for patients with hepatocellular carcinoma who progressed on sorafenib treatment (RESORCE): a randomised, double-blind, placebo-controlled, phase 3 trial. Lancet 2017;389:56-66. DOI PubMed

21. Kudo M, Finn RS, Qin S, et al. Lenvatinib versus sorafenib in first-line treatment of patients with unresectable hepatocellular carcinoma: a randomised phase 3 non-inferiority trial. Lancet 2018;391:1163-73. DOI PubMed

22. Abou-Alfa GK, Meyer T, Cheng AL, et al. Cabozantinib in patients with advanced and progressing hepatocellular carcinoma. $N$ Engl $J$ Med 2018;379:54-63. DOI PubMed PMC

23. Zhu AX, Kang YK, Yen CJ, et al; REACH-2 study investigators. Ramucirumab after sorafenib in patients with advanced hepatocellular carcinoma and increased alpha-fetoprotein concentrations (REACH-2): a randomised, double-blind, placebo-controlled, phase 3 trial. Lancet Oncol 2019;20:282-96. DOI PubMed

24. Finn RS, Qin S, Ikeda M, et al; IMbrave150 Investigators. Atezolizumab plus bevacizumab in unresectable hepatocellular carcinoma. N Engl J Med 2020;382:1894-905. DOI PubMed 